\title{
EVALUACIÓN DE LAS PROPIEDADES FISICOQUÍMICAS COMO PARÁMETROS DE CALIDAD EN LA FERMENTACIÓN DE CLONES DE CACAO CCN51, TSC01
}

\section{EVALUATION OF PHYSICOCHEMICAL PROPERTIES AS QUALITY PARAMETERS IN THE FERMENTATION OF CLONES OF COCOA CCN51, TSC01}

\author{
*Vera R. José M. ${ }^{1}$; Arrieta S. Alexandre'; Quintana Lucas F.'; García J. Alberto ${ }^{2}$ \\ *1 Servicio Nacional de Aprendizaje, SENA Centro de Atención al Sector Agropecuario Regional Santander \\ Instructor Investigador; \\ 2. Universidad Nacional Abierta y a Distancia UNAD. Docente investigador; \\ Correo electronico: imanuelvera@misena.edu.co
}

Recibido: 15 de agosto de 2017; Aprobado: 15 de noviembre de 2017

\section{RESUMEN}

La presente investigación se realizó en el municipio del playón, en la sede de Aguas Calientes, los clones de cacao evaluados correspondieron al CCN51 y TSC01 cosechados, el estudio se realizó con el fin de evaluar la calidad del proceso durante los días de la fermentación. El proceso fermentativo se realizó durante 7 días, con remoción de la masa fermentante a las 48 horas. Las mediciones se registraron en un equipo PTHr3, medidor de (temperatura, tiempo, humedad y $\mathrm{pH}$ ), efectuando registros diarios, cada media hora. Las muestras se tomaron en cosechas diferentes y fueron fermentadas 
Autor a quien dirigir la correspondencia: *Vera R. José M. Correo electronico: imanuelvera@misena.edu.co en cajones de madera, se tomaron 592 mazorcas sanas y maduras de cada clon y se obtuvieron 37 kilos de cacao en baba. Los parámetros químicos evaluados en el proceso arrojaron que el $\mathrm{pH}$ en los cotiledones no presentó variaciones marcadas, y fluctuó entre 5,1 y 5,6, En cuanto a la variación de la acidez durante el proceso de fermentación, se observó que la misma, en todos los casos evaluados, tendió a incrementarse sin mostrar cambios significativos entre días. En conclusión, las propiedades del grano seco variaron en función de los factores evaluados.

Palabras claves: fermentación, Cacao, Polifenoles, Clon.

\section{ABSTRAC}

The present investigation was carried out in the municipality of El Playón, in the headquarters of Aguas Calientes, the cacao clones evaluated corresponded to the CCN51 and TSC01 harvested, the study was carried out in order to evaluate the quality of the process during the days of the fermentation. The fermentation process was carried out for 7 days, with removal of the fermenting mass at 48 hours. The measurements were recorded on a PTHr3 device, measuring (temperature, time, humidity and $\mathrm{pH}$ ), making daily logs, every half hour. The samples were harvested differently and were fermented in wooden boxes, 592 healthy and mature cobs of each type of cocoa were taken and $37 \mathrm{~kg}$ of cocoa were obtained in drool. The chemical parameters evaluated in the process showed that the $\mathrm{pH}$ in the cotyledons did not show marked variations, and fluctuated between 5,1 and 5.6. As for the variation of the acidity during the 
fermentation process, it was observed that the same, in all cases evaluated, tended to increase without showing significant changes between days. In conclusion, the properties of the dry grain varied according to the evaluated factors.

Key words: fermentation; cacao, Poliphenol, clone.

\section{INTRODUCCIÓN}

El cultivo de cacao en Colombia es una actividad de pequeños propietarios de tierras (3.3 hectáreas, en promedio), con la participación de numerosas familias (aproximadamente 25.000) y es cultivado entre los 0 y los $1.200 \mathrm{~m}$ de altitud. El cultivo se ha adaptado a las condiciones agroecológicas de la geografía nacional, siendo la montaña santandereana la subregión natural que acoge la mayor área establecida del cultivo en el país, con aproximadamente el $50 \%$ del total nacional (FEDECACAO, 2009). Hoy en día los países sur americanos pretenden competir en el mercado mundial del cacao en calidad, apuntando a mercados de cacao fino y de aroma (González et al., 2012; Quintana et al., 2015), ya que nuestro cacao es apetecido por industrias, chocolatería gourmet y consumidores que reconocen el valor de un chocolate diferenciado por estas características, las cuales no solo dependen del proceso de fermentación y secado, como se piensa erróneamente, sino que depende desde la variedad, ambiente, tipo de suelos y manejo de la plantación, pasando por la cosecha, desgrane, fermentación, secado, almacenamiento y tostado.

La fermentación del cacao es una etapa muy importante en el procesamiento del grano, ya que se producen los cambios bioquímicos que dan origen a los precursores del aroma y del sabor. Diversos factores influyen sobre el proceso fermentativo entre ellos destaca el tipo de cacao, las condiciones ambientales, el almacenamiento de la mazorca, así como el sistema empleado en la fermentación, el tipo de fermentador, el volumen de la masa y el volteo durante el proceso. El proceso de fermentación se inicia con la transformación del azúcar de la pulpa de los granos en alcohol y dióxido de carbono; el $\mathrm{pH}$ y la temperatura se elevan. Estos cambios ocurren durante las primeras 24 o 36 horas. La actividad 
microbiana en el proceso de fermentación del cacao se ha determinado como una sucesión de etapas que en las primeras horas es afectada por levaduras, seguida de bacterias acido lácticas, las cuales disminuyen después de 48 horas de iniciado el proceso, para dar espacio a las bacterias ácido acéticas (da Veiga et al., 2013). Durante la fermentación, el etanol y el ácido acético se difunden dentro del grano, que, junto con el incremento de la temperatura, matan el embrión de la semilla y la estructura interna del grano se descompone, liberando compuestos y pigmentos que interactúan bioquímicamente con hidrolasas endógenas activadas, para desarrollar precursores del aroma y color (Afoakwa et al., 2008).

Actualmente el cacao es reconocido por su alto contenido de polifenoles y flavonoides que son saludables para el sistema cardiovascular. En el teobroma cacao I, se han detectado más de 500 compuestos volátiles (Argout et al., 2008), cuya mezcla incluyendo los alcoholes, aldehídos, ketonas, ácidos y pirazinas, desarrollados durante la fermentación, el secado, tostado y conchado generan el característico aroma del chocolate (Batista et al., 2015).

\section{MATERIALES Y METODOS}

Los frutos se cosecharon en estado de madurez en la temporada de cosecha de (Abril-Mayo); (junio-julio); (agosto septiembre), el estado de madurez de la fruta se realizó por la identificación del color del fruto, la fermentación se realizó , colectando los granos de cada clon y colocándolos en el mismo cajón, la variedad del clon TSC01, se colecto y se dispusieron en maya y se colocaron dentro del cacao en baba del CCN 51 para realizar una micro fermentación, induciendo una fermentación espontánea. La fermentación se realizó durante 7 días, dentro de los cajones de fermentación y pasado este tiempo, los granos fueron esparcidos a la casa Elba (secado), hasta alcanzar una humedad cercana al $7 \%$. Se tomaron 592 mazorcas sanas y maduras de cada tipo de cacao y se obtuvieron 37 kilos de cacao en baba.

Se extrajo una muestra de $2 \mathrm{~kg}$ de cacao fermentado y seco por cada clon, para realizar los análisis físicos en el laboratorio de evaluación sensorial del Centro de Atención al Sector Agropecuario C.A.S.A. Ubicado en Piedecuesta, Santander, se partió de una 
muestra homogénea de 100 granos de cacao, según la metodología descrita por la Norma Técnica Colombiana NTC 1252 (2003). El índice de grano se expresó como el peso promedio de un grano de cacao seco. El contenido de cascarilla se expresó como porcentaje de cascarilla. Las mediciones se registraron en un equipo PTHrS3, que se programó, para monitorear la temperatura, tiempo, humedad y $\mathrm{pH}$ diariamente, cada seis horas. Los volteos se realizaron todos los días a las 11 de la mañana. Al séptimo día, los datos fueron exportados del equipo PTHrS3 a un archivo en Excel (ver imagen 1)..

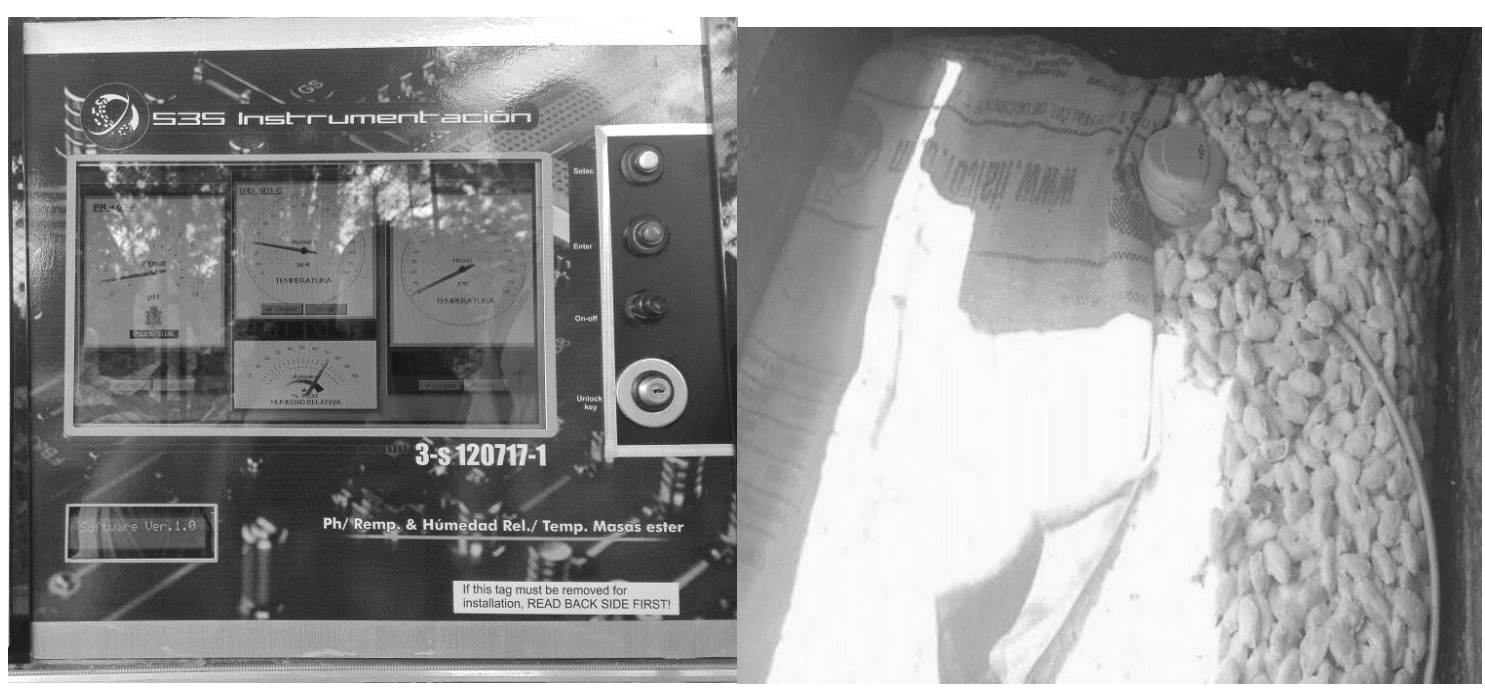

Imagen 1. Equipo PTHrS3 medición parámetros fisicoquímicos.

Los datos obtenidos en este estudio, se analizaron mediante estadística descriptiva, para identificar el comportamiento de las variables evaluadas, se realizó un análisis de comparaciones múltiples empleando el test de Fisher, en el cual se evaluó la existencia de diferencia mínima significativa (DMS), con un nivel de confianza del 95\%, se trabajó con los valores promedio de tres réplicas $(n=3)$, en base seca, y se aplicó un análisis de varianza (ANOVA), con los resultados obtenidos. 


\section{RESULTADOS.}

Los parámetros químicos evaluados en el proceso arrojaron que el pH en los cotiledones no presentó variaciones marcadas, y fluctuó entre 5,1 y 5,6 para le el CCN51, mientras que, el TSC01, el pH se situó en 5,6 y 5,78. Ver figura 1 y figura 2.

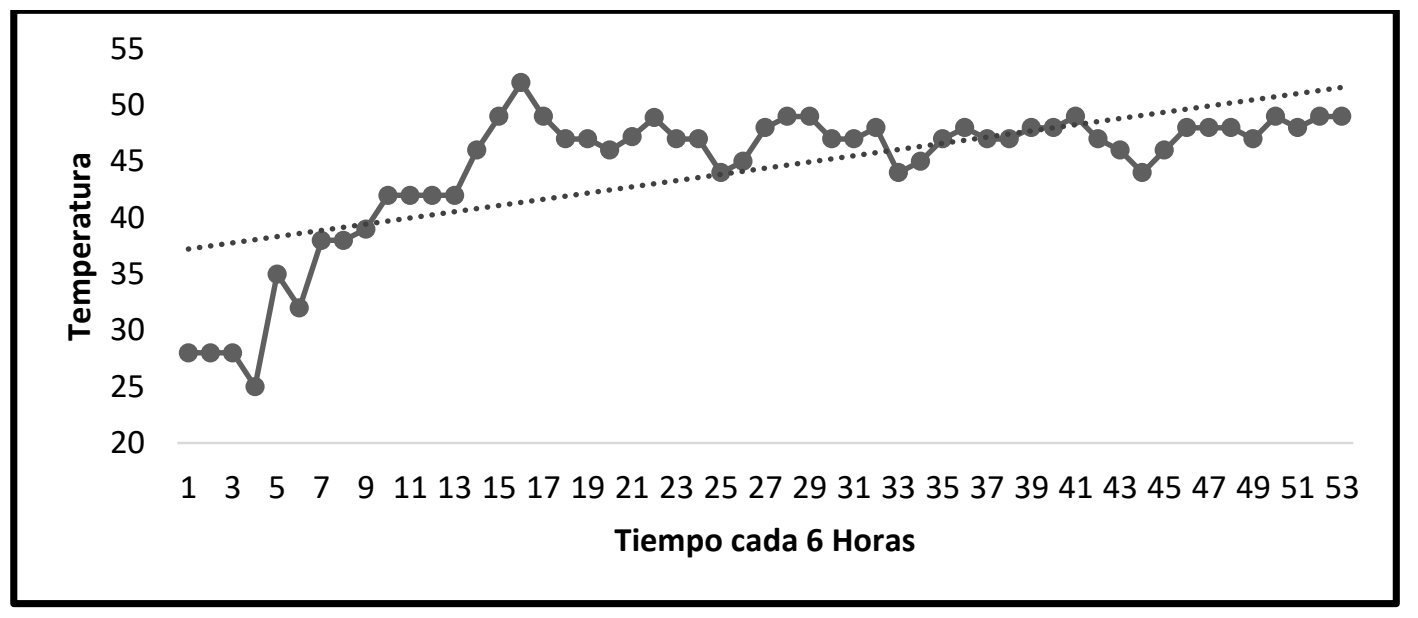

Figura 1. Comportamiento de la Temperatura Vs Tiempo en el proceso fermentativo

En la figura 1, observamos que se inicia el proceso de fermentación con una temperatura de 28 - $29^{\circ} \mathrm{C}$, luego, se observa un descenso de la misma hasta alcanzar los $25^{\circ} \mathrm{C}$, lo cual pudo ser debido al descenso de la temperatura ambiente en el sitio de la fermentación, durante las horas siguientes se presentó una tendencia al aumento de temperatura con una disminución durante los tiempos de volteo.

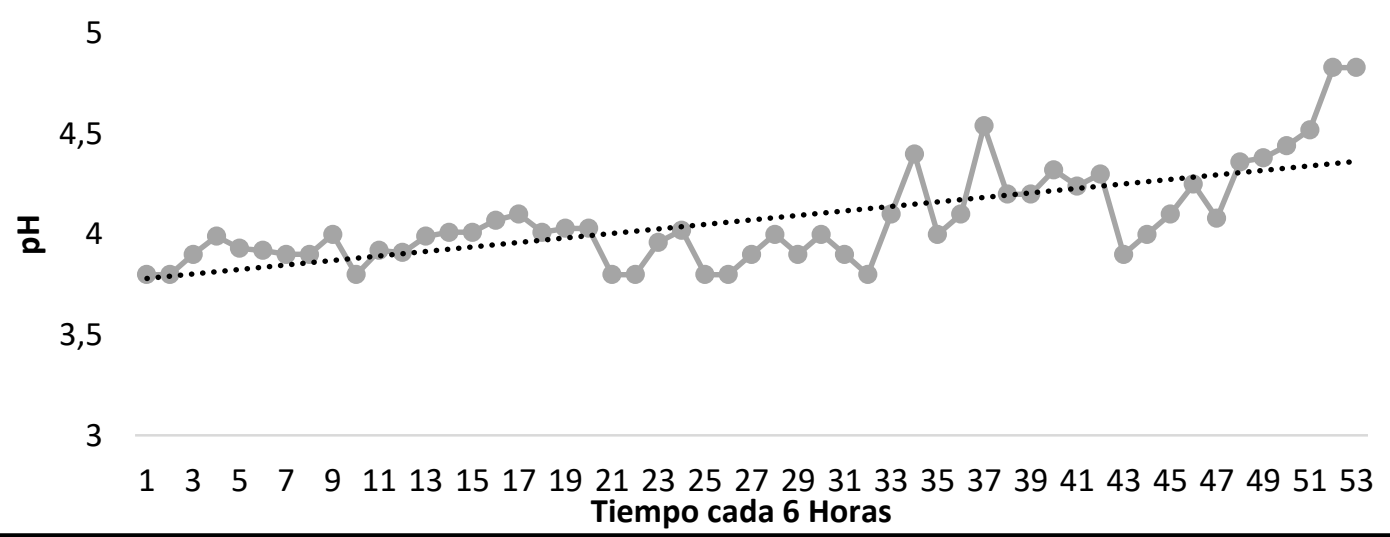

Figura 2. Comportamiento del pH Vs Tiempo en el proceso fermentativo 
En la figura 2 se observa que, durante los primeros días se presentó un descenso del pH del cotiledón, el cual fue menor en el fermentador y la acidez permaneció constante, lo cual probablemente esté relacionado con un retraso en la pérdida de la viabilidad del grano y en consecuencia de un retardo en la ruptura de las células de la pulpa y en la liberación de los jugos, lo que ocasiona un menor desarrollo de las bacterias acéticas. El descenso de la acidez en la pulpa + testa ha sido atribuido al metabolismo del ácido cítrico por acción de las levaduras y el aumento en el cotiledón a la difusión de los ácidos de la pulpa hacia esta fracción. Al 6to dial del proceso el pH del cotiledón se mantuvo con valores cercanos a 5 , considerado como indicativo de una buena calidad del grano fermentado.

Tabla 1. Características fisicoquímicas del grano de cacao CCN51

\begin{tabular}{|c|c|}
\multicolumn{1}{c|}{} & HUMEDAD \\
\hline 1. & 7,5 \\
\hline 2. & 7,4 \\
\hline 3. & 7,1 \\
\hline Promedio & 7,3 \\
\hline
\end{tabular}

INDICE DE PESO

\begin{tabular}{|c|c|}
\hline 1 & 159,8 \\
\hline 2 & 164,5 \\
\hline 3 & 166,7 \\
\hline Promedio & 163,7 \\
\hline
\end{tabular}

\begin{tabular}{|c|c|c|c|c|}
\hline \multicolumn{5}{|c|}{ PRUEBA DE CORTE } \\
\hline PRUEBA & $\begin{array}{c}\text { FERMENTADO } \\
\text { Y } 1 / 2 \\
\text { FERMENTADO }\end{array}$ & $\begin{array}{c}\text { NO } \\
\text { FERMENTADO }\end{array}$ & PISARROSOS & MOHOS \\
\hline 1 & 36 & 10 & 4 & 0 \\
\hline 2 & 41 & 7 & 2 & 0 \\
\hline 3 & 44 & 6 & 0 & 0 \\
\hline 4 & 43 & 7 & 0 & 0 \\
\hline 5 & 42 & 6 & 2 & 0 \\
\hline 6 & 46 & 4 & 0 & 0 \\
\hline
\end{tabular}

\begin{tabular}{|c|c|c|c|c|c|c|}
\hline & CESO grano & peso cotiledón & peso cascarilla & pH cotiledón & $\begin{array}{c}\mathrm{pH} \\
\text { cascarilla }\end{array}$ & $\mathrm{pH}$ mezcla \\
\hline 1 & 80,7 & 68.9 & 11.8 & 5,53 & 6,82 & 6,04 \\
\hline 2 & 80,4 & 69.0 & 10.5 & 5,54 & 6,78 & 5,92 \\
\hline 3 & 82,2 & 70.1 & 12.1 & 5,61 & 6,75 & 5,91 \\
\hline
\end{tabular}


Según los resultados obtenidos, el contenido de cascarilla presentó diferencias significativas del grano de cada genotipo evaluado, que puede ser afectada por las condiciones ambientales. Los valores medios de contenido de cascarilla evaluados en este trabajo se ubicaron dentro de los rangos de 10,5 \% a 11,2 \%, para ambos clones, estos valores coinciden con los reportados en la investigación realizada por Afoakwa et al., 2008, quienes reportan que la cascarilla representa del 10 al $11 \%$ del peso seco total del grano y el cotiledón representa la mayor parte del grano (86 - $90 \%)$.

El proceso de secado realizado en la sede aguas calientes del Playón y según los resultados obtenidos para el porcentaje de humedad, se encontró dentro de los parámetros permitidos por la NTC 1252 de 2003, con valores que oscilaron entre 7,1 y 7,5 lo cual se evidencia en la ausencia de granos infestados con moho. (Ver imagen 2).

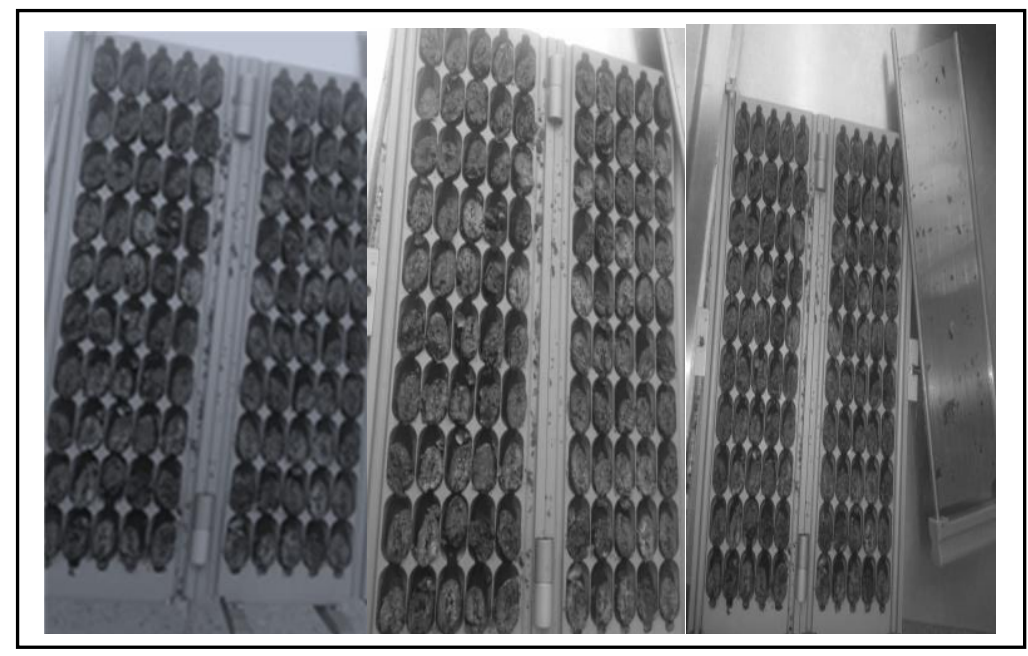

Imagen 2. Prueba de corte clon CCN51

La prueba de corte permitió identificar que en promedio el $84 \%$ de los granos analizados, presentaron una buena fermentación, observando granos cuyo proceso de fermentación fue completo, observando almendras de color marrón o chocolate, alveolos bien definidos, con formas arriñonadas, características de un buen proceso fermentativo del Cacao. 
Tabla 2. Características fisicoquímicas del grano de cacao TSC01

\begin{tabular}{|c|c|c|c|c|c|c|}
\hline \multicolumn{7}{|c|}{ PORCENTAJE DE HUMEDAD } \\
\hline 1. & 7,4 & & & & & \\
\hline 2. & 7,2 & & & & & \\
\hline 3. & 7,1 & & & & & \\
\hline Promedio & 7,26 & & & & & \\
\hline \multicolumn{2}{|c|}{ INDICE DE PESO } & & & & & \\
\hline 1 & 169 & & & & & \\
\hline 2 & 178,7 & & & & & \\
\hline 3 & 188,4 & & & & & \\
\hline Promedio & 178,7 & & & & & \\
\hline \multicolumn{5}{|c|}{ PRUEBA DE CORTE } & & \\
\hline PRUEBA & $\begin{array}{c}\text { F Y } 1 / 2 \\
\text { FERMEN }\end{array}$ & N FERMEN & PISARROSOS & MOHOS & & \\
\hline 1 & 40 & 10 & 0 & 0 & & \\
\hline 2 & 44 & 5 & 1 & 0 & & \\
\hline 3 & 44 & 6 & 0 & 0 & & \\
\hline 4 & 43 & 5 & 2 & 0 & & \\
\hline 5 & 42 & 8 & 0 & 0 & & \\
\hline 6 & 41 & 9 & 0 & 0 & & \\
\hline \multicolumn{7}{|c|}{ CASCARILLA Y COTILEDOS } \\
\hline & PESO grano & peso cotiledón & peso casca & $\mathrm{pH}$ cotiledón & $\mathrm{pH}$ cascarilla & PH mezcla \\
\hline 1 & 89,5 & 78,5 & 11 & 5,88 & 6,48 & 6,18 \\
\hline 2 & 90 & 78,6 & 11,4 & 5,76 & 6,62 & 6,14 \\
\hline 3 & 87,4 & 76,5 & 10,9 & 5,72 & 6,56 & 6,19 \\
\hline Promedio & 88,9 & 77,86 & 11,1 & 5,78 & 6,55 & 6,17 \\
\hline
\end{tabular}

En la tabla 2 se observan los resultados obtenidos de las características fisicoquímicas del grano de Cacao TSC01,encontrándose dentro de los parámetros permitidos por la NTC 1252, con un valor promedio de humedad de 7,26 \% similar al encontrado en la variedad CCN51 (7,3\%). La prueba de corte permitió identificar que en promedio el $84,6 \%$ de los granos analizados, presentaron un buen proceso de fermentativo y ausencia de mohos. 


\section{CONCLUSIÓNES}

Los parámetros químicos evaluados en el proceso arrojaron que el pH en los cotiledones no presentó variaciones marcadas, y fluctuó entre 5,1 y 5,6 tanto en los grano de cacao CCN51 como en el TSC01. En cuanto a la variación de la acidez durante el proceso de fermentación, se observó que no existe una variación significativa en todos los casos evaluados, la acidez tendió a incrementarse sin mostrar cambios significativos entre los días de fermentación. En conclusión, las propiedades del grano seco variaron en función de los factores fisicoquímicos evaluados.

\section{AGRADECIMIENTO}

Los autores agradecen al sistema de investigación e innovación y desarrollo tecnológico SENNOVA del SENA C.A.SA. Regional Santander, por el apoyo financiero brindado en la ejecución del presente proyecto, bajo el macro - proyecto, titulado: Conformación de un panel de jueces entrenados para la catación de cacao en el centro de atención al sector agropecuario. Convenio SENA-UNAD.

\section{BIBLIOGRAFIA}

Afoakwa, E. O., Paterson, A., Fowler, M., \& Ryan, A. (2008). Flavor Formation And Character In Cocoa And Chocolate: A Critical Review. Critical Reviews In Food Science And Nutrition, 48 (9), 840- 857.
Argout, X., Fouet, O., Wincker, P., Gramacho, K., Legavre, T., Sabau, X., \& Kuhn, D. (2008). Towards The Understanding Of The Cocoa Transcriptome: Production And Analysis Of An Exhaustive Dataset Of Ests Of 
Theobroma Cacao L. Generated From Various Tissues And Under Various Conditions. Bmc Genomics, 9(1), 1.

Batista, N. N., Ramos, C. L., Días, D. R., Pinheiro, A. C. M., \& Schwan, R. F. (2015). The Impact Of Yeast Starter Cultures On The Microbial Communities And Volatile Compounds In Cocoa Fermentation And The Resulting Sensory Attributes Of Chocolate. Journal Of Food Science And Technology, 1-10.

Da Veiga Moreira, I. M., Miguel, M. G. D. C. P., Duarte, W. F., Días, D. R., \& Schwan, R. F. (2013). Microbial Succession And The Dynamics Of Metabolites And Sugars During The Fermentation Of Three Different Cocoa (Theobroma Cacao L.) Hybrids. Food Research International, 54(1), 9-17

Fedecacao. (2009). Guía Técnica Para El Cultivo Del Cacao. Ministerio De Agricultura Y Desarrollo Rural. Cuarta Edición. Bogotá D.C.189 P.

González, Y., Pérez, E., \& Palomino, C. (2012). Factores Que Inciden En La Calidad Del Chocolate. Actualización En Nutrición, 13(4), 314-331.

Icontec. 2003. Cacao en Grano. NTC 1252. Bogotá D.C., Colombia.
Quintana F., Lucas F. Gómez, Salomon Garcia, Alberto y Martinez, Nubia. (2015). Perfil sensorial del clon de cacao (theobroma cacao L.) CCN51. Revista @limentech, Ciencia y Tecnología Alimentaría. ISSN: 16927125. Volumen 13 №1. Pp. 60 - 65. 